\title{
Lamb waves in binary locally resonant phononic plates with two-dimensional lattices
}

\author{
Jin-Chen Hsu and Tsung-Tsong $\mathrm{Wu}^{\mathrm{a})}$ \\ Institute of Applied Mechanics, National Taiwan University, Taipei 106, Taiwan
}

(Received 27 February 2007; accepted 19 April 2007; published online 15 May 2007)

\begin{abstract}
The authors study the propagation of Lamb waves in two-dimensional locally resonant phononic-crystal plates, composed of periodic soft rubber fillers in epoxy host with a finite thickness. Our calculations are based on the efficient plane wave expansion formulation which utilized Mindlin's plate theory. Calculated results show that the low-frequency gaps of Lamb waves are opened up by the localized resonance mechanism. The resonant frequencies of flexure-dominated plate modes are significantly dependent not only on the radius of circular rubber fillers but also on the plate thickness. The properties of localized resonance are qualitatively analogous to the vibration of a circular thin plate. (C) 2007 American Institute of Physics.
\end{abstract}

[DOI: $10.1063 / 1.2739369$ ]

Over the past decade, propagation of acoustic waves in periodic structures comprised of multicomponents has received much attention because of their renewed physical properties and potential applications. ${ }^{1-11}$ These composite materials, called phononic crystals (PCs), give rise to forbidden gaps of acoustic waves which are analogous to the band gaps for electromagnetic waves in photonic crystals. ${ }^{5}$ Major mechanisms leading to the forbidden gaps are Bragg scattering and localized resonances (LR). ${ }^{6}$ The former opens up the Bragg gap at Brillouin-zone boundaries, and the band-gap frequency corresponds to the wavelength in the order of the structural period, i.e., the lattice constant, and relates to the lattice symmetry. On the other hand, the later creates the resonant gap dictated by the frequency of resonance associated with scattering units and depends less on the lattice symmetry, orderness, and periodicity of the structure. Based on LR mechanism, Liu et $a l^{7}{ }^{7}$ demonstrated the existence of band gap at extremely low frequencies. For acoustic waves propagating in three-component PC that consists of highdensity solid core coated with a layer of soft rubber in epoxy matrix, the induced frequency gap is two orders of magnitude smaller than that of Bragg gap. ${ }^{7-10}$ Compared to the three-component PC, the LR PC with binary structure was studied by Wang et al. ${ }^{11}$ Their structure consisting of soft rubber cylinders in epoxy predicted that low-frequency gaps can also appear. Some promising applications, such as to insulate the low-frequency noise or vibration by a smallsized structure with low-frequency gaps achieved by LR PCs, were suggested.

Recently, several studies investigating the phononic lattice embedded in plate structures rather than a bulk or semiinfinite structure have been presented. ${ }^{12-15}$ These studies reveal that the propagation of Lamb waves guided in the PC plate also exhibits frequency gaps, and the ratio of plate thickness to lattice constant is also an important parameter in changing band structures. A plate structure with a forbidden gap required in the low-frequency range could also be a useful structural element in engineering. However, up to now, this part has not been studied yet.

\footnotetext{
${ }^{\text {a) }}$ Author to whom correspondence should be addressed; electronic mail: wutt@ndt.iam.ntu.edu.tw
}

In this letter, we analyze the binary LR PC plates which consist of an array of soft rubber inclusions in epoxy matrix with a finite thickness. Our calculations are based on the plane wave expansion (PWE) formulation utilizing Mindlin's theory. ${ }^{15}$ Compared to the three-dimensional PWE method ${ }^{3}$ which is difficult to cope with the guided-wave problem by taking enough PWs into account, Mindlin's theory based PWE method is very efficient in band-structure calculations for lower-order Lamb modes, and thus, band-gap calculations of thin PC plates composed of high-contrast constituents become feasible.

Schematic diagrams of a unit cell of PC plate are illustrated in Fig. 1. Figures 1(a) and 1(b) refer to the twodimensional square lattice (SL) and triangular lattice (TL), respectively. The $X Y$ plane is placed on the middle plane of the plate, and the $Z$ axis is along the thickness direction. The lattice constant and the radius of the circular fillers are denoted by $a$ and $r_{0}$, respectively. The plate thickness is $h$. In the following calculations, the density $\rho$ of soft rubber is assumed as $1300 \mathrm{~kg} / \mathrm{m}^{3}$, and that of epoxy is $1180 \mathrm{~kg} / \mathrm{m}^{3}$. The phase velocities of longitudinal and transverse waves in soft rubber are $c_{L}=33 \mathrm{~m} / \mathrm{s}$ and $c_{T}=5 \mathrm{~m} / \mathrm{s}$, respectively. ${ }^{10} \mathrm{In}$ epoxy, $c_{L}=2534 \mathrm{~m} / \mathrm{s}$ and $c_{T}=1157 \mathrm{~m} / \mathrm{s}$, respectively.

Figure 2 shows the band structure of Lamb waves in the SL rubber/epoxy plate with $r_{0}=0.4 a$ and $h=0.225 a$. These
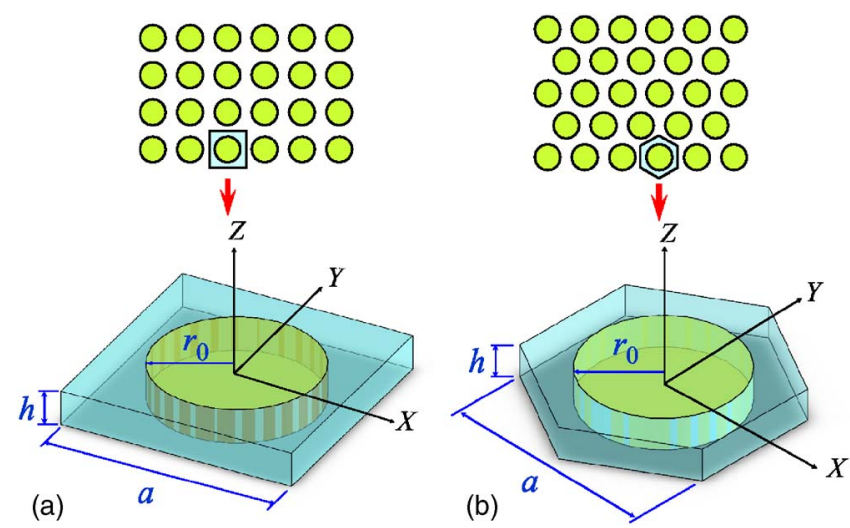

FIG. 1. (Color online) Schematic diagrams of a unit cell of PC plate with (a) SL and (b) TL, respectively. 


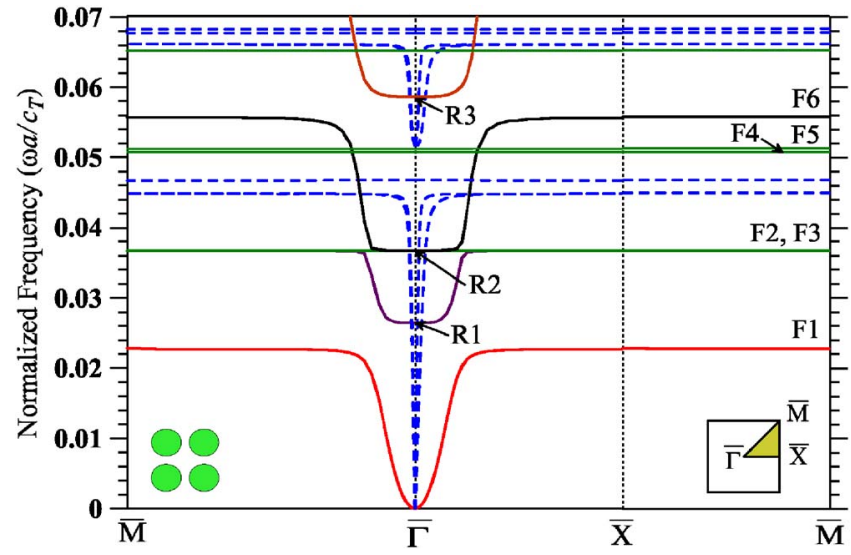

FIG. 2. (Color online) Band structure of Lamb waves in a PC plate composed of SL rubber in epoxy. $r_{0}=0.4 a$ and $h=0.225 a$.

calculations along the boundary $\bar{\Gamma}-\bar{X}-\bar{M}-\bar{\Gamma}$ of the irreducible first surface Brillouin zone employ $441 \mathrm{PWs,} \mathrm{which} \mathrm{have}$ reached a convergent result with less than $5 \%$ deviation from the test by using $1089 \mathrm{PWs}$. The frequencies are given in the normalized unit $\omega a / c_{T}$, where $c_{T}=1157 \mathrm{~m} / \mathrm{s}$. In Fig. 2 it can be observed that many flat bands exist, which indicate the resonant frequencies of localized plate modes. For such lowfrequency resonances, the band structure includes only the first three lowest-order Lamb modes without cutoff frequencies and their folding bands, while the higher-order Lamb modes with cutoff frequencies are in the much higher frequency region. These plate modes, unlike the bulk acoustic modes, are coupled modes that cannot be classified into the so-called in-plane and out-of-plane polarization modes; however, the first few flexure-dominated branches, which have a larger out-of-plane component of displacement, can be distinguished and labeled by F1-F6. These branches are sensitive to the variation of plate thickness, whereas the others, with a dominated in-plane vibration, are not. In addition, three frequencies of flexure-dominated modes with a zero group velocity near the $\bar{\Gamma}$ point, which also present a localized pattern, are denoted by $\mathrm{R} 1-\mathrm{R} 3$, respectively. Because the Lamb modes in PC plate are coupled, to find a complete band gap one has to take all polarization modes into account. As a consequence, no low-frequency gap with respect to all of the modes is found in Fig. 2. Nevertheless, the frequency bands of Lamb waves can be effectively shifted by tuning the plate thickness; it could be possible to have a complete band gap in such a low-frequency range with a different value of $h / a$. By tuning down $h$ to $0.115 a$, the calculated band structure is plotted in Fig. 3. A complete band gap is opened up in the frequency range from 0.0481 to 0.05095 which is two orders of magnitude lower than that resulting from Bragg scattering.

To further investigate the LR characteristics, the band structure referring to TL rubber/epoxy plate with $r=0.4 a$ and $h=0.115 a$ is shown in Fig. 4. Similar to the case of SL, the LR exhibiting in the behavior of flatbands and a lowfrequency gap for all modes extending the frequency range from 0.0481 to 0.0515 are also found. In addition, by comparing Fig. 3 with Fig. 4, a much more important and interesting observation is that, even though the lattice symmetry has been changed from SL into TL, the resonant frequencies keep nearly invariant with just several splittings when $r_{0}$ and $h$ remain unchanged. This is an important signature of their Downloaded 22 Dec 2008 to 140.112 .113 .225 . Redistribution subje

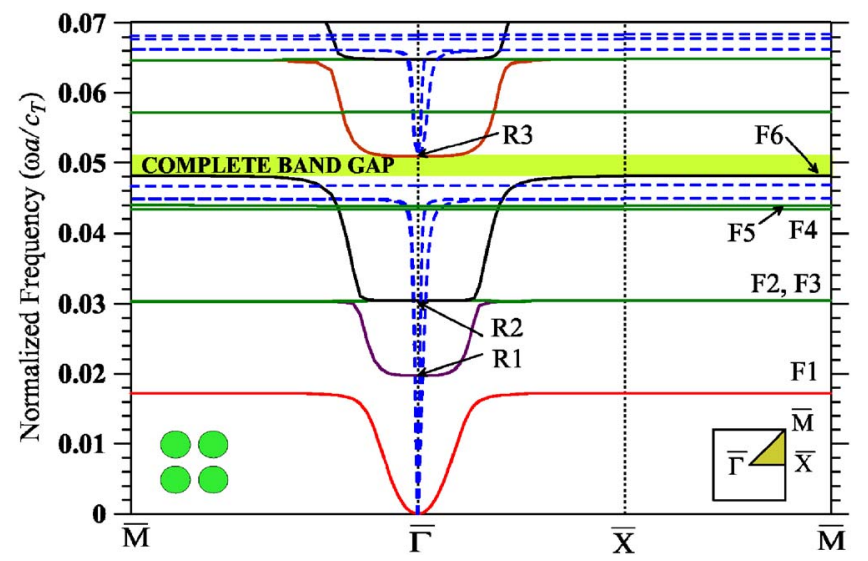

FIG. 3. (Color online) Band structure of Lamb waves in a PC plate composed of SL rubber in epoxy. $r_{0}=0.4 a$ and $h=0.115 a$.

resonant nature. Particularly, consider the upper and bottom edges of the complete band gaps appearing as $h=0.115 a$. The bottom-edge frequencies of the gaps are the same for both cases of SL and TL, so the bottom-edge frequencies relate to the resonance localized inside the rubber, where the associated eigenfrequencies are determined by the oscillation of rubber only but irrelevant to lattice symmetries. For the upper-edge frequencies of the gaps, the value is slightly shifted from 0.05095 to 0.0515 as the SL is changed into TL. This implies that the frequencies of upper edges correspond to the resonant modes where hosting material epoxy is also involved and the neighboring rubber fillers are weakly coupled, therefore, the lattice symmetries can affect the resonance. This suggests that the LR and low-frequency gap will also appear in heterogeneous plates without lattice symmetry or periodicity. It is worth noting that the transmission spectrum, which is unobtainable by this PWE formulation, is important for actual experiments of LR plates. To go further, other methods, such as multiple-scattering theory ${ }^{5,6,13}$ and finite-difference time-domain method, ${ }^{4,8}$ should be suitable to be applied to the theoretical analysis of transmission properties of LR PC plates.

In order to give a physical insight, a simplified continuum model-flexural vibration of a circular thin plate with a thickness $h$ and radius $r_{0}$-is introduced to explain the LR characteristics of PC plate. The wave equation for the thin plate is ${ }^{16}$

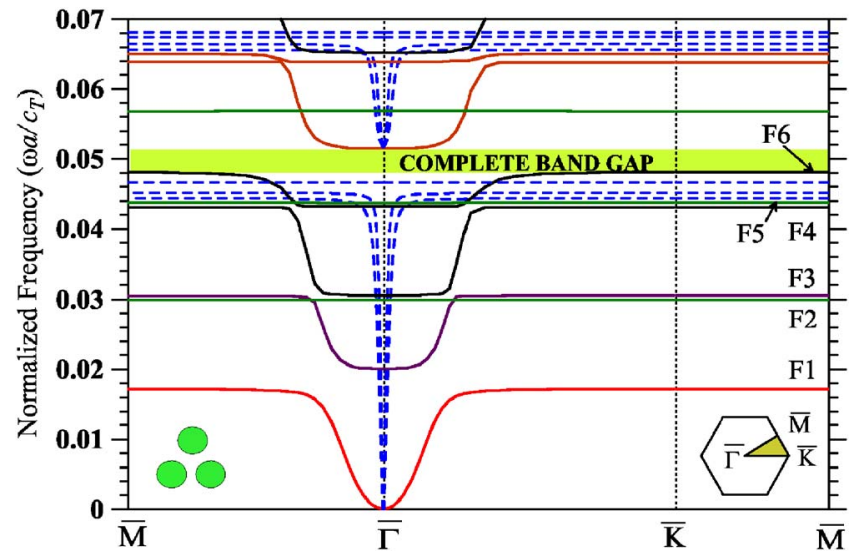

FIG. 4. (Color online) Band structure of Lamb waves in a PC plate composed of TL rubber in epoxy. $r_{0}=0.4 a$ and $h=0.115 a$.

to AIP license or copyright; see http://apl.aip.org/apl/copyright.jsp 

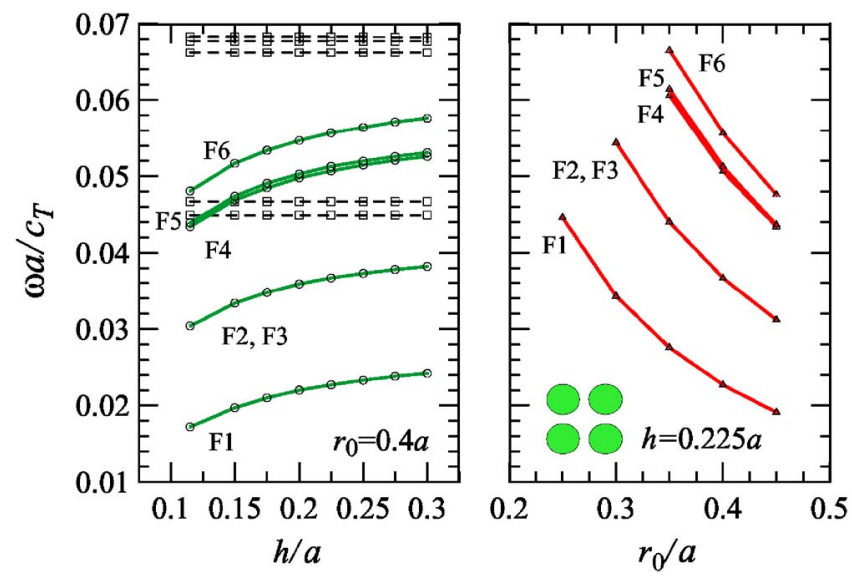

FIG. 5. (Color online) (a) Thickness dependence of resonant frequencies in SL with $r_{0}=0.4 a$. F1-F6 modes (solid lines) and modes with dominated in-plane vibrations (dashed lines). (b) Variations of resonant frequencies F1-F6 in SL with $h=0.225 a$ as a function of $r_{0} / a$.

$$
\frac{E h^{3}}{12\left(1-\nu^{2}\right)} \nabla^{4} u_{Z}+\rho h \frac{\partial^{2} u_{Z}}{\partial t^{2}}=0,
$$

where $E$ and $\nu$ are Young's modulus and Poisson's ratio, respectively, and $u_{Z}$ is the flexural displacement. In the PC plate, though the rubber fillers are surrounded by epoxy which is not perfectly rigid, the displacements taking place at the rubber-epoxy interfaces are much smaller than that in the interiors of rubber fillers because of the high contrast between their elastic stiffness. To simplify our discussion, the edges of the circular thin plate are assumed to be clamped. Equation (1) with the clamped boundary conditions then leads to following relation:

$$
\beta_{\mathrm{nm}}^{4}=\frac{12 \rho \omega_{\mathrm{nm}}^{2} r_{0}^{4}\left(1-\nu^{2}\right)}{E h^{2}},
$$

where $\beta_{\mathrm{nm}}$ is an eigenvalue associated to a specific normal mode of the plate, and $\omega_{\mathrm{nm}}$ is the corresponding eigenfrequency. This relation qualitatively reveals the dependence between resonant frequency and geometrical parameters. In other words, for a particular resonant mode, the resonant frequency increases as $h$ is increased; on the other hand, it decreases when $r_{0}$ becomes larger.

To further show that the observed resonances are localized, Fig. 5 demonstrates the dependences of the resonance frequencies on $h$ and $r_{0}$. Figure 5(a) illustrates the frequency variations of the resonant modes in the SL rubber/epoxy PC plate as a function of $h / a$, where $r_{0}=0.4 a$. The resonant frequencies of the modes with dominated in-plane vibrations (dashed lines) remain constants as $h / a$ changes; this exhibits that these resonance modes are not at all sensitive to the change of $h / a$. In contrast, the resonant frequencies of those flexure-dominated modes (solid lines) are gradually increased as $h / a$ increases. On the other hand, Fig. 5(b) shows the $r_{0} / a$ dependence of resonance frequencies in the SL plate with $h=0.225 a$. Clearly, in Fig. 5(b) the resonant frequencies of F1-F6 modes decrease as $r_{0}$ increases, and the decreases are relatively more rapid. The above geometrical-dependence properties of resonant frequency agree with the anticipations given by Eq. (2), which correspond to the fact that the resonances of the PC plate are localized in the rubber fillers.

To summarize, we have studied the propagation of Lamb waves in the LR PC plate composed of rubber fillers in epoxy. Numerical calculations show that the resonant frequencies of the flexure-dominated modes significantly depend not only on the radius of the rubber inclusions but also on the thickness of the composite plate. These properties can be explained by the vibration of a circular thin plate. By tuning the $h / a$ ratio properly, complete band gaps in an extremely low-frequency range are found to exist. The LR PC plates are also suggested to serve as promising elements in the vibration shielding of plate structures.

The authors gratefully acknowledge the financial support from the National Science Council of Taiwan (Grant No. NSC 95-2221-E-002-223).

${ }^{1}$ M. S. Kushwaha, P. Halevi, L. Dobrzynski, and B. Djafari-Rouhani, Phys. Rev. Lett. 71, 2022 (1993).

${ }^{2}$ M. M. Sigalas and E. N. Economou, J. Sound Vib. 158, 377 (1992).

${ }^{3}$ T.-T. Wu, Z.-C. Hsu, and Z.-G. Huang, Phys. Rev. B 71, 064303 (2005).

${ }^{4}$ T.-T. Wu, C.-H. Hsu, and J.-H. Sun, Appl. Phys. Lett. 89, 171912 (2006).

${ }^{5}$ A. Modinos, N. Stefanou, I. E. Psarobas, and V. Yannopapas, Physica B 296, 167 (2001).

${ }^{6}$ Z. Liu, C. T. Chan, and P. Sheng, Phys. Rev. B 65, 165116 (2002).

${ }^{7}$ Z. Liu, X. Zhang, Y. Mao, Y. Y. Zhu, Z. Yang, C. T. Chan, and P. Sheng, Science 289, 1734 (2000).

${ }^{8}$ C. Goffaux, J. Sánchez-Dehesa, A. Levy Yeyati, Ph. Lambin, A. Khelif, J. O. Vasseur, and B. Djafari-Rouhani, Phys. Rev. Lett. 88, 225502 (2002).

${ }^{9}$ M. Hirsekorn, Appl. Phys. Lett. 84, 3364 (2004).

${ }^{10}$ C. Goffaux and J. Sánchez-Dehesa, Phys. Rev. B 67, 144301 (2003).

${ }^{11}$ G. Wang, X. Wen, J. Wen, L. Shao, and Y. Liu, Phys. Rev. Lett. 93, 154302 (2004).

${ }^{12}$ X. Zhang, T. Jackson, E. Lafond, P. Deymier, and J. Vasseur, Appl. Phys. Lett. 88, 063118 (2006).

${ }^{13}$ R. Sainidou and N. Stefanou, Phys. Rev. B 73, 184301 (2006).

${ }^{14}$ A. Khelif, B. Aoubiza, S. Mohammadi, A. Adibi, and V. Laude, Phys. Rev. E 74, 046610 (2006).

${ }^{15}$ J.-C. Hsu and T.-T. Wu, Phys. Rev. B 74, 144303 (2006).

${ }^{16}$ P. M. Morse, Vibration and Sound (McGraw-Hill, New York, 1948), Chap. V, Sec. 21, pp. 208-211. 